Supporting Information for

\title{
Quantitative Understanding of Energy Transfer between Fluorescent Proteins Connected via Flexible Peptide Linkers
}

Toon H. Evers, Elisabeth M.W.M. van Dongen, Alex C. Faesen, E.W. Meijer and Maarten Merkx

Laboratory of Macromolecular and Organic Chemistry, Department of Biomedical Engineering, Eindhoven University of Technology, P.O. Box 513, 5600 MB Eindhoven, The Netherlands 


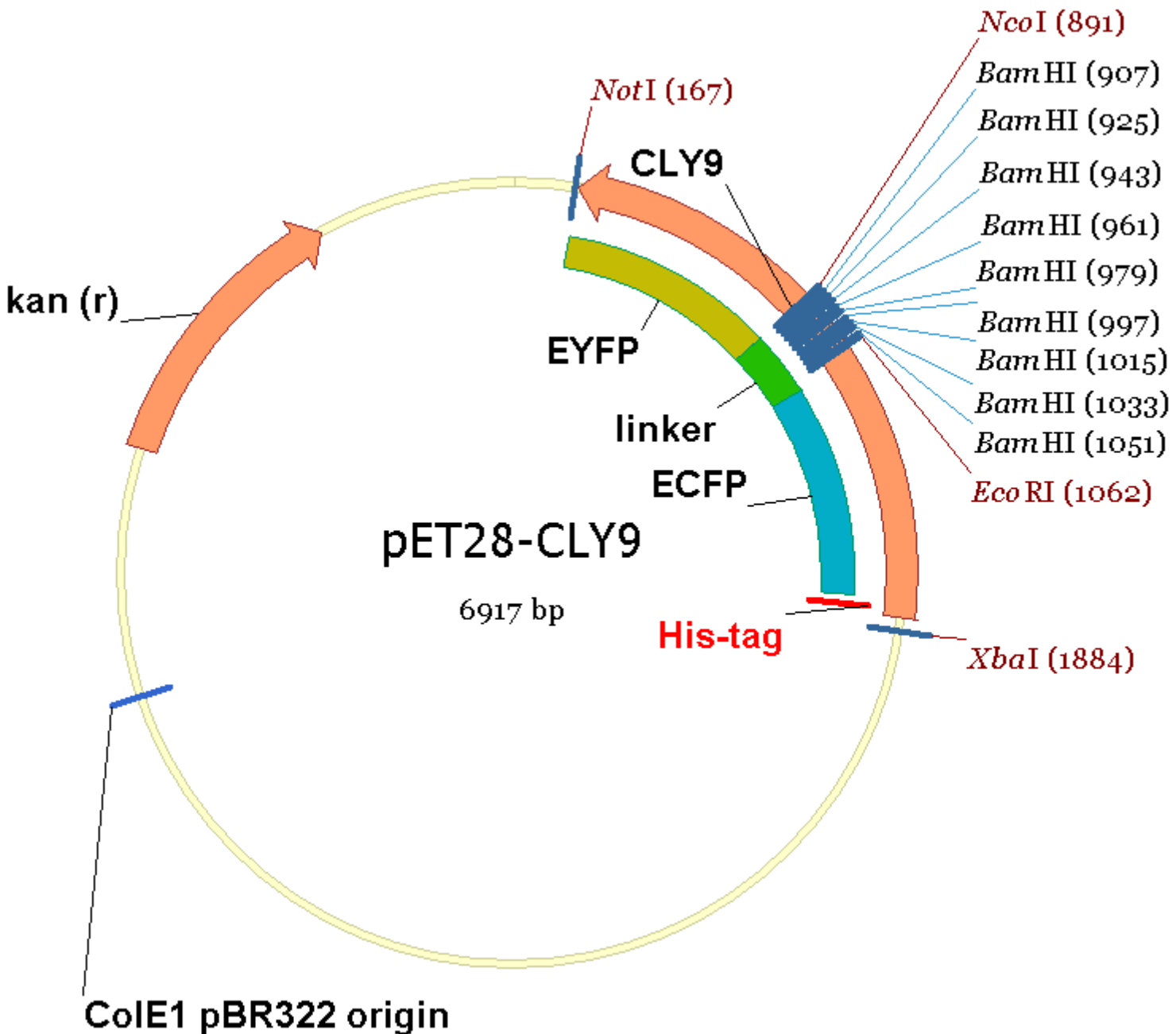

Figure S1. Vector map of pET28-CLY9, indicating the open reading frame of CLY9 and various other features. 
- - - - - - - - - - - - - - - - - - - - - - - MetGlySerSerHisHisHisHisHisHi TCTAGAAATAATTTTGTTTAACTTTAAGAAGGAGATATACTATGGGCAGCAGCCATCATCATCATCATCA $X b a$ I start

s.SerSerGlyLeuValProArgGlySerHi sMetValserLysGlyGluGluLeuPheThrGlyValVal CAGCAGCGGCCTGGTGCCGCGCGGCAGCCATATGGTGAGCAAGGGCGAGGAGCTGTTCACCGGGGTGGTG

ProIleLeuValGluLeuAspGlyAspValAsnGlyHisLysPheSerValSerGlyGluGlyGluGlyA CCCATCCTGGTCGAGCTGGACGGCGACGTAAACGGCCACAAGTTCAGCGTGTCCGGCGAGGGCGAGGGCG

spAlaThrTyrGlyLysLeuThrLeuLysPheIleCysThrThrGlyLysLeuProValProTrpProTh ATGCCACCTACGGCAAGCTGACCCTGAAGTTCATCTGCACCACCGGCAAGCTGCCCGTGCCCTGGCCCAC

rLeuValThrThrLeuThrTrpGlyValGlnCysPheSerArgTyrProAspHisMetLysGlnHisAsp CCTCGTGACCACCCTGACCTGGGGCGTGCAGTGCTTCAGCCGCTACCCCGACCACATGAAGCAGCACGAC

PhePheLysSerAlaMetProGluGlyTyrValGlnGluArgThrIlePhePheLysAspAspGlyAsnT TTCTTCAAGTCCGCCATGCCCGAAGGCTACGTCCAGGAGCGCACCATCTTCTTCAAGGACGACGGCAACT

yrLysThrArgAlaGluVal Lys PheGluGlyAspThrLeuValAsnArgIleGluLeuLysGlyIleAs ACAAGACCCGCGCCGAGGTGAAGTTCGAGGGCGACACCCTGGTGAACCGCATCGAGCTGAAGGGCATCGA

pPheLysGluAspGlyAsnIleLeuGlyHisLysLeuGluTyrAsnTyrIleSerHisAsnValTyrIle CTTCAAGGAGGACGGCAACATCCTGGGGCACAAGCTGGAGTACAACTACATCAGCCACAACGTCTATATC

ThrAlaAspLysGlnLysAsnGlyIleLysAlaAsnPheLysI leArgHisAsnIleGluAspGlyserV ACCGCCGACAAGCAGAAGAACGGCATCAAGGCCAACTTCAAGATCCGCCACAACATCGAGGACGGCAGCG

alGlnLeuAlaAspHisTyrGlnGlnAsnThrProIleGlyAspGlyProValLeuLeuProAspAsnHi TGCAGCTCGCCGACCACTACCAGCAGAACACCCCCATCGGCGACGGCCCCGTGCTGCTGCCCGACAACCA

STyrLeuSerThrGlnSerAlaLeuSerLysAspProAsnGluLysArgAspHisMetValLeuLeuGlu CTACCTGAGCACCCAGTCCGCCCTGAGCAAAGACCCCAACGAGAAGCGCGATCACATGGTCCTGCTGGAG

PheValThrAlaAlaGlyIleThrLeuGlyMetAspGluLeuTyrLysSerGlyIleArgGlyGlyserG TTCGTGACCGCCGCCGGGATCACTCTCGGCATGGACGAGCTGTACAAGTCCGGAATTCGTGGTGGATCCG ECOR I BAmH I

lyGlySerGlyGlySerGlyGlySerGlyGlySerGlyGlySerGlyGlySerGlyGlySerGlyGlySe GTGGATCAGGTGGATCCGGTGGTAGTGGTGGATCCGGAGGTTCTGGTGGATCCGGTGGTTCAGGTGGATC

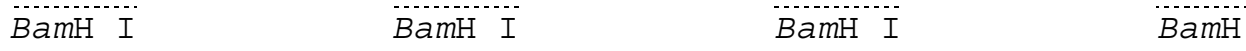

rGlyGlySerGlyGlySerGlyGlySerGlyGlySerGlyGlySerGlyGlySerGlyGlySerGlyGly CGGTGGATCTGGTGGATCCGGTGGTAGTGGTGGATCCGGTGGATCTGGTGGATCCGGTGGTAGTGGTGGA I

SerGlyGlySerThrMetValSerLysGlyGluGluLeuPheThrGlyValValProlleLeuValGluL TCCGGAGGTAGCACCATGGTGAGCAAGGGCGAGGAGCTGTTCACCGGGGTGGTGCCCATCCTGGTCGAGC $\mathrm{H} I$ NCO I 
euAspGlyAspValAsnGlyHisLys PheSerValSerGlyGluGlyGluGlyAspAlaThrTyrGlyLy TGGACGGCGACGTAAACGGCCACAAGTTCAGCGTGTCCGGCGAGGGCGAGGGCGATGCCACCTACGGCAA

SLeuThrLeuLys PheIleCysThrThrGlyLysLeuProVal ProTrpProThrLeuValThrThrPhe GCTGACCCTGAAGTTCATCTGCACCACCGGCAAGCTGCCCGTGCCCTGGCCCACCCTCGTGACCACCTTC

GlyTyrGlyLeuGlnCysPheAlaArgTyrProAspHisMetLysGlnHisAspPhePheLysSerAlaM GGCTACGGCCTGCAGTGCTTCGCCCGCTACCCCGACCACATGAAGCAGCACGACTTCTTCAAGTCCGCCA

etProGluGlyTyrValGlnGluArgThrIlePhePheLysAspAspGlyAsnTyrLysThrArgAlaGl TGCCCGAAGGCTACGTCCAGGAGCGCACCATCTTCTTCAAGGACGACGGCAACTACAAGACCCGCGCCGA

uValLys PheGluGlyAspThrLeuValAsnArgIleGluLeuLysGlyIleAspPheLysGluAspGly GGTGAAGTTCGAGGGCGACACCCTGGTGAACCGCATCGAGCTGAAGGGCATCGACTTCAAGGAGGACGGC

AsnIleLeuGlyHisLysLeuGluTyrAsnTyrAsnSerHisAsnValTyrIleMetAlaAspLysGlnL AACATCCTGGGGCACAAGCTGGAGTACAACTACAACAGCCACAACGTCTATATCATGGCCGACAAGCAGA

ysAsnGlyIleLysValAsnPheLysIleArgHisAsnIleGluAspGlySerValGlnLeuAlaAspHi AGAACGGCATCAAGGTGAACTTCAAGATCCGCCACAACATCGAGGACGGCAGCGTGCAGCTCGCCGACCA

STyrGlnGlnAsnThrProIleGlyAspGlyProValLeuLeuProAspAsnHisTyrLeuSerTyrGln CTACCAGCAGAACACCCCCATCGGCGACGGCCCCGTGCTGCTGCCCGACAACCACTACCTGAGCTACCAG

SerAlaLeuSerLysAspProAsnGluLysArgAspHisMetValLeuLeuGluPheValThrAlaAlaG TCCGCCCTGAGCAAAGACCCCAACGAGAAGCGCGATCACATGGTCCTGCTGGAGTTCGTGACCGCCGCCG

lyIleThrLeuGlyMetAspGluLeuTyrLys* * * . . - . - . -

GGATCACTCTCGGCATGGACGAGCTGTACAAGTAAAGCGGCCGC stop Not I

Figure S2. The DNA sequence between the $X b a$ I and Not I sites that differs from pET$28 \mathrm{a}(+)$ and the amino acid translation of the open reading frame of CLY9 are depicted below. Important features are underlined (His-tag, red; ECFP, blue; linker, green; EYFP, yellow). 

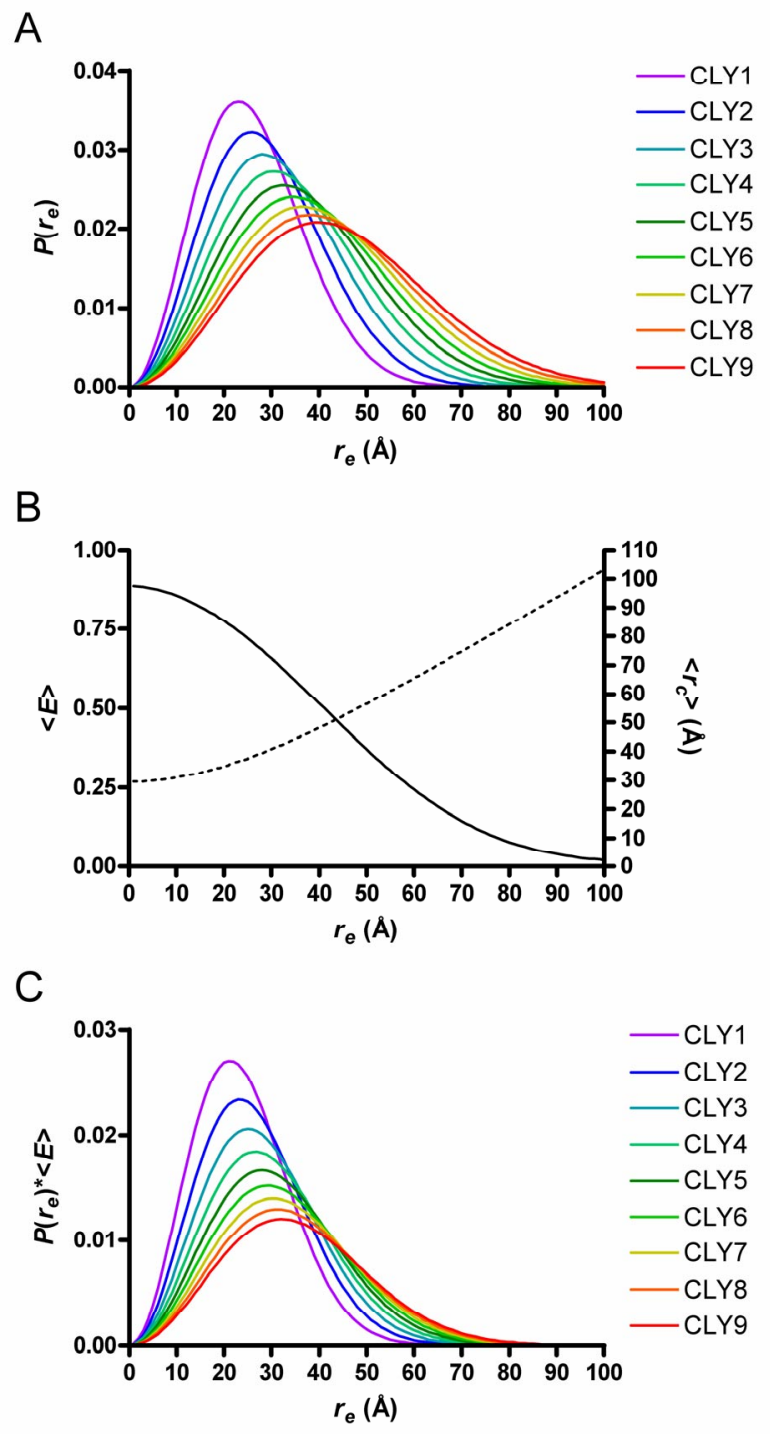

Figure S3: Modeling the energy transfer efficiency for CLYx using the Gaussian chain model to describe the peptide linker. (A) Distribution functions showing the probability $P\left(r_{e}\right)$ for each end-to-end distance of the peptide linker for CLY1-9 calculated using the Gaussian chain model assuming a characteristic ratio of 2.3. (B) Plot showing the average energy transfer $\left(\langle E\rangle\right.$, solid line) and the average interchromophore distance $\left(\left\langle r_{c}\right\rangle\right.$, dashed line) as a function of $r_{e}$. (C) The contribution of each $r_{e}$ to the overall energy transfer $\langle E\rangle_{\text {ensemble }}$, calculated by multiplying the probability of each $r_{e}$ with the corresponding $\langle E\rangle$. 